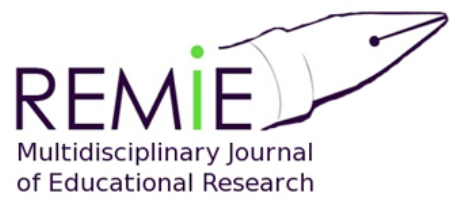

Instructions for authors, subscriptions and further details:

http://remie.hipatiapress.com

\title{
Educación Inclusiva del Alumnado Inmigrante: Lecciones desde Estados Unidos, Canadá y Australia
}

Diana Valero ${ }^{1}$, Teresa Plaja Viñas ${ }^{2}$

1) Universidad de Zaragoza. España

2) Universidad de Barcelona. España

Date of publication: June $15^{\text {th }}, 2017$

Edition period: October 2017- February 2018

To cite this article: Valero, D., Plaja Viñas, T. (2017). Educación Inclusiva del Alumnado Inmigrante: Lecciones desde Estados Unidos, Canadá y Australia. Multidisciplinary Journal of Educational Research, 7(3), 316- 338. doi: 10.17583/remie.2017.2946

To link this article: http://dx.doi.org/10.17583/remie.2017.2946

\section{PLEASE SCROLL DOWN FOR ARTICLE}

The terms and conditions of use are related to the Open Journal System and to Creative Commons Attribution License (CC-BY). 


\section{Educación Inclusiva del Alumnado Inmigrante: Lecciones desde Estados Unidos, Canadá y Australia}

Diana Valero

Universidad de Zaragoza
Teresa Plaja Viñas

Universidad de Barcelona

\section{Resumen}

Muchos programas, en España o Europa, entienden al alumnado inmigrante como un colectivo homogéneo, aplicando soluciones iguales a todos, sin lograr promover su inclusión socio-educativa. Sin embargo, algunos países anglosajones observan diferencias en este grupo que influyen en su éxito educativo e inclusión social y cuyo conocimiento puede ayudar a promover actuaciones que atiendan mejor a esta población. El artículo describe y analiza el concepto de estudiante con escolaridad formal limitada o interrumpida y discute los factores que la escuela tiene en cuenta para fomentar su inclusión socio-educativa, abogando por una intervención holística que tenga en cuenta este alumnado necesita mucho más que centrar su aprendizaje únicamente el nuevo idioma, por ejemplo el apoyo social para superar los problemas socioemocionales que les acompañan. Resulta especialmente relevante para España y Europa, pues recientemente acuden cada vez más alumnos y alumnas con estas características a nuestras escuelas.

Palabras clave: estudiante bilingüe, estudiantes de minorías culturales, diversidad del alumnado, escuelas inclusivas 


\section{Inclusive Immigrant Student Education: Lessons from the United States, Canada and Australia}

Diana Valero

Universidad de Zaragoza
Teresa Plaja Viñas

Universidad de Barcelona

\section{Abstract}

Many programs, in Spain or Europe, understand immigrant students as a homogeneous group, applying equal solutions to all, without being able to promote their socio-educational inclusion. However, some Anglophone countries observe differences within this group that may influence their educational success and social inclusion and whose knowledge can help to promote actions that better serve this population. The article describes and analyzes the concept of a student with limited or interrupted formal education and discusses the factors that schools may consider for promoting their socio-educational inclusion, advocating a holistic intervention that takes into account this students' needs which go beyond focusing their learning only on the new language, for example social support to overcome the socioemotional problems that accompany them. It is especially relevant for Spain and Europe, as more and more students with these characteristics are arriving to our schools.

Keywords: bilingual student, minority group students, student diversity, inclusive schools 


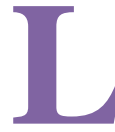

a crisis humanitaria que Europa está viviendo en sus fronteras debido a la guerra y los cientos de miles de personas desplazadas, pueden suponer la llegada de menores, regular o irregularmente, a las aulas de las escuelas europeas. Pero dado lo reciente del fenómeno, todavía no conocemos las características de esta población, ni los factores que la escuela debe tener en cuenta a la hora de fomentar el éxito educativo y la inclusión social de este alumnado inmigrante.

En este sentido, la mayor parte de países sigue entendiendo al grupo de alumnado inmigrante como un grupo homogéneo, tal y como Menken, Klein y Chaef (2012) señalan, "es particularmente urgente la necesidad de comprender mejor la diversidad de los bilingües emergentes en secundaria y la realidad que estos alumnos y alumnas tienen, ya que las necesidades educativas son diferentes; en cambio, este grupo es a menudo mal percibido como monolítico, tanto en la academia como en el entorno escolar" (p.122), hecho también expuesto por Aubert, Molina, Shubert yVidu (2017).

El objetivo de este artículo es tomar como referencia las investigaciones previas sobre la heterogeneidad del grupo de alumnado inmigrante con el fin de informar las políticas educativas con este colectivo, especialmente dado que el reto al que se enfrentan los países europeos.

Partiendo de esta necesidad de conocer las diferencias dentro del grupo de alumnado inmigrante, desde hace ya una década, los países angloparlantes, especialmente Estados Unidos, Canadá y Australia, han comenzado a investigar sobre las diferentes experiencias dentro del grupo de alumnado inmigrante. La literatura en este campo se centra en Estados Unidos (Boyston \& Short, 2003; Klein \& Martohardjono, 2008; Ruíz de Velasco, Fix \& Chu, 2000;), Canadá (Thomas, 2004; Toohey \& Derwing, 2008) y Australia (Matthews, 2008; Medina Audelo,2017; Woods, 2009) por lo que el idioma que se utiliza como referencia es el inglés, y se parte de que todos los y las estudiantes inmigrantes lo desconocen. Los tres grupos principales de alumnos y alumnas, siguiendo a Olsen y Jaramillo (1999) y Freeman, Freeman y Mercuri (2002), son:

1. Recién llegados con una escolarización adecuada.

2. Estudiantes de inglés de largo plazo.

3. Estudiantes con escolaridad formal interrumpida. 
En el presente artículo nos centramos en el último grupo, los y las estudiantes con escolaridad formal limitada o interrumpida, en adelante SLIFEs, ya que se trata de la situación que van a vivir las y los menores refugiados que llegan a las aulas europeas y que ya caracteriza a muchos otros y otras estudiantes dentro de nuestros sistemas educativos. Los SLIFEs se caracterizan porque, además de las condiciones del resto del alumnado inmigrante (como el desconocimiento de la lengua de instrucción), han sufrido interrupciones en su educación, generalmente en sus países de origen y, debido a esta interrupción, se encuentran al menos dos cursos por debajo de sus iguales. Hasta ahora se consideraba que los y las estudiantes con escolaridad formal interrumpida eran generalmente refugiados/as (Crandall \& Greenblatt, 1998), ya que entre 1980 y 1990 fueron muchas personas las que acudieron a USA; pero actualmente este colectivo se está ampliando más allá de esta característica incluyendo a otros grupos. Además, esta caracterización incluye al alumnado refugiado, pero no hay que olvidar que el concepto "refugiado" es una calificación jurídica $y$, por tanto, podemos tener alumnado que, aunque no lo sea formalmente, tenga las mismas características y, además, no pueda disfrutar del amparo que supone ser considerado legalmente como refugiado. Por ello es preciso una categorización más amplia que nos permita entender las características de este alumnado, cumplan o no con los requisitos jurídicos para adquirir la condición de refugiado e independientemente de la condición administrativa en la que se encuentren. Para ello, en el presente artículo se realiza un análisis teórico sobre las características de este alumnado y las dificultades que los y las educadoras están abordando en el aula con el fin de adelantarnos a una situación que puede llegar a ser frecuente en los centros educativos españoles.

Se presenta inicialmente una definición de lo que son los y las estudiantes con escolaridad formal limitada o interrumpida y los escasos datos existentes sobre su presencia en las aulas. Posteriormente, se abordan sus características académicas y socioemocionales ya que, como se concluye, se requiere un enfoque holístico para la atención de un alumnado con necesidades específicas, que pueden desbordar a los profesores y profesoras por su falta de formación específica. 


\section{Metodología}

Se ha llevado a cabo un análisis bibliográfico en las principales bases de datos educativas, especialmente ERIC (Educational Resources Information Center) y JCR (Journal Citation Report) en base a los descriptores: estudiante con escolaridad formal interrumpida (SIFE), estudiante con escolaridad formal limitada o interrumpida (SLIFE), estudiante inmigrante, estudiante de inglés (ELL) y estudiante refugiado.

\section{Los Estudiantes con Escolaridad Formal Interrumpida (SLIFEs): Factores Académicos}

Para definir a los SLIFEs existen múltiples nomenclaturas "escolaridad formal limitada" (Menken, 2010), "escolaridad previa limitada" (Freeman, Freeman \& Mercuri, 2002), "recién llegados" (Short, 2002) o "Underschooled", término utilizado por Olsen y Jaramanillo (1999) para identificar a los y las estudiantes que "vienen a nosotros [USA] en Secundaria, que nunca han asistido a la escuela en su país natal; o pueden tener uno o incluso varios años de instrucción inadecuada. Otros pueden tener importantes lagunas en su educación, al haber asistido a la escuela sólo esporádicamente" (Olsen \& Jaramanillo, 1999, p. 228). Pero como Wright (2010) denuncia al mencionar las etiquetas que se utilizan para referirse al estudiantado que ha pasado mucho tiempo aprendiendo inglés (Long Term English Language Learners), estas nomenclaturas pueden sugerir un déficit en los propios alumnos y alumnas ya que en ninguna parte de la "etiqueta" se indica que los y las estudiantes estén aprendiendo activamente. En este sentido se están produciendo nuevas investigaciones para evitar esta perpetuación promoviendo el uso del término "bilingües emergentes" (Por ejemplo: García, Klein \& Falchi, 2008; o Menken, 2013), centradas en los elementos positivos y fortalezas de este alumnado. Los y las estudiantes bilingües emergentes son niños y niñas inmigrantes o hijos e hijas de inmigrantes, o pueblos indígenas, que están añadiendo la lengua de instrucción a la lengua que utilizan en casa, y durante el proceso educativo ellos mismos se convierten en bilingües (García 2009). Esta es la realidad de la mayoría de los países, en donde prácticamente la totalidad de 
alumnado inmigrante podría ser bilingüe emergente. En este sentido, como García et. al (2008) explican, es "a través de la escuela y por medio de la adquisición del inglés, que estos infantes se convierten en bilingües, capaces de seguir funcionando en su lengua materna, así como en inglés, su nuevo idioma y el de la escuela" (García et. al, 2008, p. 6).

Desde el punto de vista de la Administración y de muchas investigaciones, los y las estudiantes SLIFEs se caracterizan por una escolaridad formal previa limitada o nula, lo que conlleva una alfabetización en la lengua materna deficiente y la falta de dominio de la lengua de instrucción (Boyson \& Short, 2003; Freeman et. al, 2002; Klein \& Martohardjono, 2008; Ruiz de Velasco et. al, 2000).

En esta misma línea, una de las primeras definiciones que se dieron es la propuesta por Advocates for Children (2010), que describe a los SLIFEs como estudiantes que, además de venir de un país cuyo idioma no es inglés:

1. Entraron a los Estados Unidos después del segundo grado;

2. Han tenido por lo menos dos años menos de escolaridad que sus pares;

3. Funcionan al menos dos años por debajo del nivel esperado en lectura y matemáticas; y

4. Pueden estar pre-alfabetizados en su lengua materna.

Por tanto, los SLIFE se caracterizan por tener una alfabetización inexistente o limitada en su lengua materna, lo que supone que su rendimiento académico esté muy por debajo del nivel que les correspondería por edad (Freeman et. al, 2002; Klein \& Martohardjono, 2008; Ruíz de Velasco et. al, 2000).

En el caso de Canadá se da incluso un paso más allá estableciendo tres categorías de estudiantes SLIFEs en función de su alfabetización previa: prealbafetos y analfabetos; semi-alfabetos; y funcionalmente alfabetizados (Johansson et al 2001).

Las limitaciones en su lengua materna se deben a la interrupción en su educación, que en algunos casos puede incluso implicar no haber acudido nunca a la escuela. En este sentido, la literatura se ha hecho eco de las motivaciones que han dado lugar a la interrupción de la educación o a la limitación de ésta. Lee (2012) señala que los SLIFEs pueden provenir de los países donde la pobreza, los desastres y disturbios civiles afectan el 
desarrollo de la alfabetización y oportunidades para la educación. Hickey (2015) por su parte profundiza en este aspecto y hace referencia a que "las razones de la falta de acceso a la educación formal de los estudiantes pueden ir desde el conflicto armado y el desplazamiento como refugiados hasta haber crecido en las zonas rurales con instalaciones escolares limitadas y altos costes para uniformes o materiales escolares" (Hickey, 2015, p. 81). A estas razones, DeCapua y Marshall (2010) añaden que su limitación en la educación a menudo se debe a la falta de recursos, maestros poco capacitados o el tipo de educación recibida. Respecto a este último aspecto, la educación recibida en el país de origen, Boyson y Short (2003) hacen referencia a que, aunque no haya habido una interrupción propiamente, puede haberse dado una educación limitada o que esta educación haya sido muy diferente. Es lo que Klein y Martohardjono (2006) han señalado como "non-parallel-schooling", es decir, educación en sus países de origen que no equivale a la que hubieran recibido en el país de acogida.

En este sentido, la descripción más amplia ha sido la dada por DeCapua y Marshall (2011), quienes describen a estos estudiantes en tres formas:

1. Pueden provenir de países en los que no tiene escolarización obligatoria, por lo tanto, no tienen conocimiento de la escuela. Por ejemplo, ellos pueden no saber cómo sostener un lápiz o sentarse en una silla. Puede que no hayan desarrollado un lenguaje escrito, o el lenguaje escrito es un fenómeno relativamente nuevo.

2. Pueden comenzar la escuela en un lugar y continuamente moverse, como es el caso de muchos hijos e hijas de los y las trabajadoras migrantes. En este caso, las ausencias prolongadas y cambios en las escuelas son una característica común.

3. Es posible que hayan asistido a la escuela con regularidad en sus países de origen, pero la calidad de la educación que recibieron fue significativamente menor que la que hubieran recibido en Estados Unidos, por lo que están varios años por detrás de sus pares estadounidenses. Esta falta de educación de calidad puede ser debida a la insuficiencia de recursos, la escasez de maestros capacitados, la falta de fondos de los padres para pagar la escuela, 
las condiciones climáticas que interrumpen la escolarización (como es el caso de algunos estudiantes indios cuyas escuelas cierran durante la temporada de lluvia), o el tipo de educación que recibieron.

Es decir, estos estudiantes provienen de familias diversas en términos de etnia, religión, origen del lenguaje, dominio del inglés, estatus migratorio, y la clase social.

Dentro de las características del alumnado SLIFE está la edad en la que se considera que un estudiante es SLIFE. En este aspecto tampoco hay un acuerdo, por ejemplo Calderón (2007) categoriza SLIFES como estudiantes entre grado 4 y 12 (desde $4^{\circ}$ de Primaria a $2^{\circ}$ de Bachiller); sin embargo, el estudiantado que por diferentes motivos no recibió los 3 primeros años de educación primaria también podría considerarse que tiene una escolaridad formal interrumpida; en este sentido Advocates for Children ampliaba la definición a todos aquellos que llegaron después de $2^{\circ}$ grado. $\mathrm{Y}$ aunque durante la etapa de educación primaria la interrupción es muy importante, la situación se vuelve más complicada para el estudiantado que llega al país de acogida durante la educación secundaria. Esto se debe a que, además de necesitar dominar la lengua de instrucción para acceder a contenidos más complejos, la necesitan para comunicar estos nuevos conocimientos (Bunch, 2010).

Además, los SLIFEs que llegan al país de acogida durante la educación secundaria han perdido cursos durante la educación primaria, lo que implica que han recibido muchas menos horas de clases de lengua y matemáticas, lo que dificulta la adquisición de la lengua de instrucción y de conocimientos complejos. Por ejemplo, tomando como referencia los últimos datos del informe "Education at Glance" (OECD, 2015) en educación primaria, la media de la OCDE es dedicar el $27 \%$ de las horas de clase a asignaturas relacionadas con las habilidades linguísticas y el 17\% con las matemáticas. El tiempo que se dedica a estas asignaturas en secundaria se reduce a un $16 \%$ y un $13 \%$ respectivamente por suponer que se han adquirido los conocimientos de alfabetización básicos. Un alumno o alumna que ha perdido 2 años de escolarización, habría perdido 427.14 horas de clases de lengua si tomamos como referencia la media de la OCDE. Esta cifra podría 
elevarse a 560 horas si tomamos por ejemplo el caso de México (OECD, 2015)

Junto con la falta de horas, que afecta a estudiantes que han podido no sufrir interrupciones, el informe desarrollado por la Oficina de Inmigrantes de Indiana señala que los SLIFEs pueden no ser capaces de leer y escribir en su lengua materna, y también pueden carecer de una comprensión de los conceptos básicos y conocimiento de los contenidos y las habilidades de pensamiento crítico que sus compañeros ya dominan (Indiana Department of Education-Office of English Language Learning and Migrant Education, 2008, p. 1).

Por otra parte, no se debe olvidar que, junto al menor número de horas y las características del alumnado, un estudio de García (1999) sobre la ciudad de New York, señalaba que el profesorado de estos alumnos y alumnas en sus países de origen habían tenido una escolarización formal limitada.

Puede parecer un grupo muy específico y no representativo dentro del grupo de alumnado inmigrante, pero ya en un estudio llevado a cabo en 1993 se estimaba que al menos el 20\% del estudiantado con un nivel dominio del inglés limitado de High School habían perdido dos años o más de escolarización (Fleishman \& Hospstock, 1993). En este mismo sentido, en 1999 una investigación cualitativa con profesorado de California señaló que, según los propios profesores y profesoras, al menos el $15 \%$ de sus estudiantes de Middle School habían perdido dos o más años de escolaridad desde los 6 años de edad (Olsen, Jaramanillo, MCcall-Perez \& White, 1999, p. 170).

A pesar de que no han sido ampliamente estudiados, existen otros aspectos de los estudiantes SLIFEs que se deben tener en cuenta y que pueden afectar a sus resultados académicos como son la situación socioemocional y familiar, un aspecto que se aborda a continuación. 


\section{Factores Socioemocionales y Familiares}

Pocos estudios han abordado esta situación de los y las estudiantes SLIFEs, y los que lo han hecho ha sido de manera descriptiva y poco profunda, pero nos dejan importantes factores a tener en cuenta. Específicamente son tres las áreas en las que se podría agrupar la revisión de la literatura: el estrés derivado de su falta de experiencia en la educación formal, así como sus dificultades académicas, las experiencias traumáticas que vivieron en sus países de origen y en el viaje a USA y las actuales condiciones de vida en USA.

El primer factor, que además es extensible a otros muchos estudiantes de inglés como L2, es el que resulta más ampliamente estudiado dentro de las escasas investigaciones llevadas a cabo en este ámbito. En el informe del centro de estudiantes inmigrantes de Indiana (2008) se hace referencia al estrés y la frustración que pueden sufrir estos estudiantes. La educación formal de un estudiante puede haber sido interrumpida por la migración, la guerra, la falta de acceso a la educación, y/o circunstancias socioeconómicas o culturales (Indiana, 2008, p. 1). Como resultado, el estudiante puede sufrir un trastorno de estrés post-traumático, puede estar bajo la angustia grave, o puede ser completamente abrumado por la necesidad de asimilar un nuevo entorno escolar en un nuevo idioma.

También puede suceder que un SLIFE esté muy emocionado por asistir a la escuela regularmente, y puede tener grandes esperanzas en su capacidad para trabajar y mantener a su familia. Sin embargo, el descubrimiento de que se encuentra muy por detrás de sus compañeros puede ser una fuente de gran frustración. Por mucho que mejore siempre está persiguiendo un objetivo que se aleja de él ya que sus compañeros continúan aprendiendo contenidos mientras él debe adquirir también el inglés. La comprensión de que el cumplimiento de sus objetivos será más difícil de lo esperado puede ser devastadora.

En este mismo sentido se orienta la investigación llevada a cabo en NYC (Advocates for Children, 2010) y que señala que "aparte de la integración en el entorno escolar, casi todos los y las estudiantes tenían necesidades emocionales intensas derivadas de sus retos académicos. Mientras que la investigación indica que los SLIFEs entran a las escuelas de 
Nueva York con expectativas muy altas, incluyendo planes para obtener un diploma de escuela secundaria, pronto se desaniman. Casi la totalidad del estudiantado con el que trabajamos se sintió aislado en la escuela, avergonzado por estar tan lejos detrás de sus compañeros, consciente de su falta de progreso, y abrumado por la lucha para ajustar la configuración y las actividades escolares. En general, la escuela era un lugar alienante donde tenían pocas o ninguna experiencia con éxito" (Advocates for Children, 2010, p. 26).

Por ejemplo, el trabajo de la Dra. Birman (2007) señala que muchos menores refugiados han sufrido eventos muy traumáticos. Muchos han vivido bombardeos, han escapado de los soldados, han huido de sus hogares de repente bajo una gran amenaza, o han sido testigos de asesinatos y otros actos terribles, o incluso ellos han participado. Los trabajadores de los campos de refugiados generalmente dejan los campamentos de refugiados durante la noche, y los campos pueden ser muy inseguros con incursiones nocturnas. También puede suceder que la comida no sea suficiente. Finalmente, los muchos movimientos que los niños y niñas han vivido, incluyendo el traslado a los Estados Unidos, pueden ser a la vez emocionantes y traumáticos.

Las familias se separan durante esta transición, y su reasentamiento en los barrios urbanos de Estados Unidos puede ser muy decepcionante y estresante. Para los infantes, algunas de las cosas que pasan a su alrededor puede desencadenar los recuerdos traumáticos, o pueden reaccionar de maneras que ellos han aprendido a reaccionar con el fin de protegerse a sí mismos. En este mismo sentido, "cabe destacarse que es común para el SLIFE con quien trabajamos haber sufrido trauma significativo en sus países de origen, durante la migración, y después de llegar a los EE.UU. Estos menores y adultos jóvenes se propusieron encontrar parientes u otras conexiones en Nueva York, llegaron solos y solas o pronto se encontraron a solas, y terminaron sin hogar o en el cuidado del sistema de crianza. Algunos fueron matriculados por primera vez en la escuela por los trabajadores sociales de servicios sociales, pero la continua inestabilidad en sus situaciones de vida sólo se añade al trauma que ya habían experimentado, lo que hace aún más difícil satisfacer sus necesidades emocionales" (Advocates for Children, 2010, p. 26). 
Respecto al segundo factor señalado, las experiencias traumáticas que vivieron en sus países de origen y en el viaje a USA, Birman (2007) destaca que el profesorado debe anticipar y ayudar a los infantes a orientar los eventos que les pueden estar perturbando; darles seguridad cuando aparezca algún contenido traumático y escucharles activamente y ofrecerles apoyo. Aunque puntualiza también que no se les debe forzar a probar esos hechos ya que muchas veces es imposible y les generaría un estrés innecesario.

Kanu (2008), haciendo referencia explícitamente a los estudiantes SLIFEs, que además son refugiados, señala que también se pueden ver afectados por el estrés causado por la aculturación, numerosos retos psicosociales y la posibilidad de haber experimentado traumas.

El estudiantado que tiene estas experiencias se encuentra representado en todos los niveles del espectro de la alfabetización. Sin embargo, la interrupción traumática que han experimentado es significativamente distinta de lo que habría sido típico para su desarrollo. Los comportamientos que van desde la impulsividad a la hipervigilancia no son inusuales porque los y las estudiantes han vivido o están viviendo en un entorno turbulento y responden a los traumas de diferentes formas (Craig, 2008).

Finalmente el tercer factor, su situación actual en USA. Carhill, Gayatan y Suárez-Orozco (2007) consideran que la inmigración es una de las transiciones más estresantes que una familia puede sufrir, señalan que los cambios en los contextos, las relaciones y los roles pueden ser desorientadores, dar lugar a una sensación de pérdida, y pueden tener un impacto duradero en el desarrollo de los niños y niñas. En este sentido, la velocidad de un infante para absorber conocimientos en la nueva cultura puede crear oportunidades o causar tensiones (Carhill, Gayatan \& SuárezOrozco, 2007).

Los distintos factores emocionales (y cómo éstos se refuerzan en las interacciones sociales) son claves para la adquisición de segundas lenguas. Lightbown y Spada (2006) especifican que las características de la personalidad, tales como la inhibición, la ansiedad, la autoestima, la dominación, la locuacidad y capacidad de respuesta tienen una influencia importante en el éxito en el aprendizaje de idiomas. Señalan concretamente que la inhibición desalienta la toma de riesgos, la cual es necesaria para el 
progreso en el aprendizaje de idiomas. Además, debido a que la educación formal de un estudiante puede haber sido interrumpida por la migración, la falta de acceso a la educación, o de otras circunstancias, los SLIFEs pueden sufrir angustia por la necesidad de asimilarse a un nuevo entorno escolar (Robertson \& Lafond, 2008). Estos sentimientos de preocupación, nerviosismo o estrés juegan un papel importante en el aprendizaje de un segundo idioma, interfiriendo con el proceso de aprendizaje (Lightbown \& Spada, 2006).

En este sentido, el entorno resulta de una gran influencia en los factores emocionales del alumnado a la hora de enfrentarse a la adquisición de una segunda lengua (Lightbown \& Spada, 2006), especialmente la comunidad en la que se desenvuelven los SLIFEs. Así, estos mismos autores (Lightbown \& Spada, 2006) defienden que el que este alumnado tenga referentes adultos en su comunidad, que hablan esta segunda lengua de instrucción, puede ser un factor motivador, porque quieren funcionar como nativos o llegar a ser como las personas que hablan el idioma, o bien tener el objetivo práctico de poder comunicarse con ellas.

En el caso del alumnado inmigrante, y especialmente el recién llegado, como son los SLIFEs, el aprendizaje de la nueva lengua es esencial. De hecho, como señala Abedi (2008), las oportunidades de aprendizaje están íntimamente vinculadas al dominio de la lengua de instrucción. En este sentido, como ya se ha visto, el proceso de adquisición de la lengua de instrucción por parte del alumnado inmigrante está influido por el contexto y las oportunidades de aprender que éste le proporciona, tanto en casa, como en la escuela y en otros espacios de socialización. Así, uno de los elementos claves que condicionan las oportunidades de aprendizaje es la motivación que se desprende de las interacciones que éstos mantienen con todo tipo de personas. (Lightbown \& Spada, 2011).

A continuación se describen otras de las características de este alumnado y que tienen influencia en su rendimiento académico, ya que influyen en su manera de aprender, y que están relacionadas fundamentalmente con factores culturales. 


\section{Factores Psicosociales}

La Oficina de estudiantes inmigrantes de Indiana describió algunas de las características fundamentales que les afectan: el estrés, la frustración, los déficits académicos y de alfabetización y el alto riesgo de abandono escolar (Indiana, 2008:1). En este caso se puede ver cómo, además de las necesidades académicas y socioemocionales ya señaladas, se hace referencia a la consecuencia, el alto riesgo de abandono. Algunos autores vinculan su tendencia a abandonar los estudios con su percepción (o la de sus padres) de la falta de retribución a corto plazo, es decir, no sienten que la educación vaya a suponer un cambio significativo y mejoría de su situación (Ruíz de Velasco, Fix \& Chu Clewell, 2000).

Específicamente para los SLIFEs se considera que tienen un alto riesgo de abandonar la escuela secundaria dado que su desconocimiento del idioma provoca una menor implicación con la institución educativa: "los estudiantes latinos en riesgo, de entre 16-19 años, que consideraban que no hablaban bien inglés, eran cuatro veces más propensos a abandonar la escuela secundaria que sus compañeros que hablaban bien inglés". (Fry, 2003 citado en Spaulding, Carolino \& Amén, 2004, p.8)

Pero estos aspectos responden únicamente a sus características desde un punto de vista académico, siendo la situación y complejidad de los estudiantes inmigrantes mucho más amplia. Así, además de la situación académica, algunos autores se han hecho eco de otro tipo de retos en el trabajo con estos estudiantes. Por ejemplo, Brown, Miller y Mitchell (2006) señalan las alfabetizaciones culturales, que permiten entender conceptos concretos, y la pedagogía de trabajo ya que algunos pueden no estar acostumbrados, por ejemplo, al trabajo en grupos o lo contrario.

En este mismo sentido, DeCapua y Marshall (2010) señalan la importancia de tener en cuenta los procesos de aprendizaje de estos estudiantes, ya que por cuestiones culturales pueden no ser los mismos que los de la educación que van a recibir en las escuelas americanas, lo que requiere un proceso de ajuste. Entre estos elementos destacan cómo el aprendizaje se logra de manera compartida (responsabilidad de grupo) y como el aprendizaje es un proceso oral (DeCapua \& Marshall, 2010, p. 166; Tellado, 2017). 
DeCapua y Marshall (2010) van más allá y explican las características del aprendizaje de estos estudiantes incluyendo en ellas la perspectiva cultural, haciendo referencia a que las condiciones de aprendizaje de los y las estudiantes SLIFEs pasan por la relevancia inmediata y el aprendizaje como una forma de relacionarse con los demás.

En esta misma línea, Kanu (2008), señala entre las dificultades académicas de los SLIFEs la velocidad de los curriculums, la escasa diferenciación entre la educación para SLIFEs y la educación general cuando están en clases regulares, dificultades para tomar apuntes o escribir textos académicos, pensamiento crítico y habilidades de organización.

Esta situación representa un desafío para los educadores, ya que por un lado, este alumnado requiere instrucción en los conceptos y las habilidades necesarias para el éxito académico, incluyendo cómo estudiar y tomar notas, y cómo participar en las discusiones en clase básicas. Por otra parte, su éxito académico se basa en el cumplimiento de las normas cada vez más sofisticadas y evaluaciones en inglés. Así que, incluso cuando los estudiantes no dominan el inglés o no son educados formalmente, en lugar de centrarse exclusivamente en la base y/o habilidades de recuperación, es importante ofrecer lecciones diseñadas para desarrollar el conocimiento crítico, con contenidos que reflejen vida de los estudiantes, los intereses y cultura (Clough, 2017; DiCerbo \& Loop, 2003).

Tener a los estudiantes con una amplia gama de habilidades de lectura y escritura en una misma aula, puede ser abrumador para el profesorado, sobre todo los nuevos maestros o maestras que no han sido debidamente capacitados para atender las necesidades de esta población de estudiantes.

Otros indicadores de la población SLIFE pueden incluir: registros educativos pobres, ausencia de registros educativos, o expedientes educativos con importantes lagunas; auto-informe o informes de los padres de ausencia de la escuela por largos períodos de tiempo; pobres registros de asistencia a las anteriores escuelas; notas pobres; comprensión muy débil de contenido académico; y, bajo rendimiento en las pruebas estandarizadas (DeCapua et al., 2007:42). Además, la ausencia de mecanismos de identificación de este colectivo (Ruíz de Velasco et. al, 2000) ha hecho que se pierdan en el sistema y que en la elaboración de planes para alumnos y alumnas inmigrantes se asuma un cierto nivel de habilidades básicas en vez 
de diseñar programas que las desarrollen que deberían haberse asumido en la educación elemental (Crandall \& Greenblat, 1998; Crandall et al, 1998; García 1999; Mace-Matluck, Alexander-Kasparik \& Queen, 1998).

La mayoría de las investigaciones que se han llevado a cabo en este sentido han dado una visión de los y las estudiantes SLIFEs como carentes, basada en dos dificultades principales: alfabetización nula o limitada y necesidad de aprender la lengua de instrucción (por ejemplo: Boyson y Short, 2003; Freeman et. al, 2002; Klein \& Martohardjono, 2008; Ruíz de Velasco et. al, 2000 ). Menken es muy crítica con los estudios que se limitan a estos dos aspectos y denuncia que "esta visión de los y las estudiantes SLIFEs como, de alguna manera, mentalmente limitados únicamente porque no tienen altos nivel de lenguaje o alfabetización para los objetivos académicos es una extrapolación problemática" (Menken, 2013 , p. 453). Hay que tener en cuenta que, junto con estas necesidades, los estudiantes inmigrantes en general y SLIFEs en particular traen a la nueva escuela grandes fortalezas y potencialidades que pueden servir de palanca para su aprendizaje, como es su resiliencia, su alta motivación, su conocimiento práctico y su riqueza intercultural (Hickey, 2015, p. 82), lo que puede utilizarse como fuente de apoyo para desarrollar políticas adecuadas para ayudar en su formación.

\section{Conclusiones y Recomendaciones}

Aunque el trabajo presentado se basa en la situación de Estados Unidos, debemos tomar nota de este caso y adelantarnos a un fenómeno que cada vez resulta más evidente en España: el grupo de alumnado inmigrante no es homogéneo.

Si la mayoría de los estudiantes inmigrantes tienen la dificultad de que no hablan la lengua de instrucción y tienen una mayor tendencia al fracaso escolar y al abandono de la escuela (Flecha, Soler-Gallart \& Sordé, 2015; Girbés-Peco, Macías \& Álvarez, 2015), los estudiantes SLIFEs tienen, además, la dificultad de que su escolarización se vio interrumpida por diferentes causas. Por ello, junto con la necesidad de adquirir un nuevo idioma y nuevos contenidos propios de su edad, deben rellenar las lagunas académicas previas, adquirir nuevos hábitos escolares e incluso en algunos 
casos llevar a cabo una alfabetización que hasta ahora no han tenido posibilidad de recibir. Todo ello imbuido de una situación socioemocional y socioeconómica muy vulnerable y que influye en sus resultados educativos, llevando a las aulas situaciones que el profesorado no está preparado para atender.

A pesar de que las Administraciones han adoptado políticas activas, no se está alcanzando el éxito académico de este colectivo, por lo que se encuentran en riesgo de exclusión educativa y social. No hablan la lengua de instrucción ni tienen un buen nivel educativo y, en consecuencia, se enfrentan a enormes desafíos que, sin los apoyos necesarios, son difíciles de superar, lo que con frecuencia conduce al fracaso y al abandono escolar. Esta situación supondría para estos jóvenes su exclusión educativa, lo que lleva a un alto riesgo de exclusión social dado que cada vez se necesita una mayor cualificación para acceder al mercado laboral.

En su preocupación por ellos, la mayor parte de las investigaciones se han centrado en sus necesidades académicas, aunque han dejado al margen otras necesidades que afectan muy directamente a su rendimiento escolar y que deben ser tenidas en cuenta para alcanzar el desarrollo de programas educativos de éxito, como son las necesidades socioemocionales y factores culturales.

Cabe también señalar que las potencialidades y fortalezas de estos estudiantes todavía pasan desapercibidas para una parte de la comunidad educativa, cuando es esencial tenerlas en cuenta en el desarrollo de nuevos programas. En este sentido, los y las estudiantes inmigrantes pueden, si se les da la oportunidad, compensar sus deficiencias académicas (interrupciones, mala calidad, falta de rutinas y habilidades) con su bagaje cultural y linguiístico. Además, su conocimiento práctico puede aprovecharse para lograr el éxito académico en el país de acogida, llegando a ser bilingües y a alcanzar el nivel deseado de educación, aunque puede costarles más tiempo que a sus compañeros y compañeras.

Pero, con el fin de aprovechar esta riqueza en el aula es imprescindible conocer sus antecedentes y poner en práctica actuaciones educativas que se ha demostrado que tienen éxito con toda la población en general y con colectivos con características similares en particular, como serían en este caso los resultados que se obtienen a través de las comunidades de 
aprendizaje, los grupos interactivos o las tertulias literarias dialógicas, entre otras. En este sentido, este estudio ha tratado de dar a conocer en profundidad las características de los estudiantes con escolaridad formal limitada o interrumpida que pueden servir de apoyo para permitir elaborar propuestas educativas adecuadas a sus necesidades.

Por todo ello, coincidimos con las conclusiones a las que han llegado estudios similares señalando que "la preparación de estos estudiantes para una participación efectiva en la sociedad en general requiere de una educación integral, basada en estándares que tomando como punto de partida su conocimiento práctico, consistente y sensible, promueva el dominio de las habilidades académicas, la alfabetización, el aprendizaje socio-emocional, y la conciencia cultural" (Nysed, 2011, p. 3). Es decir, apostamos por un enfoque holístico que atienda a todos los ámbitos del estudiante y que, basándose en evidencias científicas, promueva el máximo de aprendizaje para el alumnado SLIFE.

Para España y Europa. conocer las características y potencialidades de este alumnado supondría la posibilidad de crear políticas y programas más enfocados y basados en evidencias, lo que permitiría desarrollar el derecho a la educación de este alumnado y promover su inclusión social.

\section{Referencias Bibliográficas}

Aubert, A., Molina, S., Shubert, T., \& Vidu, A. (2017). Learning and inclusivity via interactive groups in early childhood education and care in the hope school Spain. Learning, Culture and Social Interaction, 13(1), pp. 90-103. doi:10.1016/j.lcsi.2017.03.002

Abedi, J. (2008) Classification system for English language learners: Issues and recommendations. Educational Measurement: Issues \& Practice, 27(3), pp. 17-31. doi:10.1111/j.1745-3992.2008.00125.x Advocates for children (2010). Students with interrupted formal education: A challenge for the New York City public schools. Retrieved from http://www.advocatesforchildren.org/sites/default/files/library/sife_2010. $\mathrm{pdf} ? \mathrm{pt}=1$

Birman, D. (2007). Refugee children with low literacy skills or interrupted education: Identifying challenges and strategies. Denver, CO: Spring 
334 Valero \& Plaja - Educación Inclusiva del Alumnado Inmigrante

Institute. Retrieved from

https://www.springinstitute.org/Files/refugeechildrenbehavior3.pdf

Boyson, B. A., \& Short, D. (2003). Secondary school newcomer programs

in the United States (No. 12). Center for Research on Education,

Washington DC; Center for Applied Linguistics. Retrieved from

http://citeseerx.ist.psu.edu/viewdoc/download?doi=10.1.1.488.91\&rep=re

p1\&type $=$ pdf

Brown, J., Miller, J., \& Mitchell, J. (2006). Interrupted schooling and the acquisition of literacy: Experiences of Sudanese refugees in Victorian secondary schools. Australian Journal of Language and Literacy, 29(2), pp. 150-162.

Bunch, G. C. (2010). Preparing mainstream secondary content-area teachers to facilitate English language learners' development of academic language. Yearbook of the National Society for the Study of Education, 109(2), pp. 351-383.

Calderon, M. (2007). Teaching reading to English language learners, grades 6-12: A framework for improving achievement in the content areas (ed.). Thousand Oaks, CA: Corwin Press.

Carhill, A., Gayatan, F., \& Suárez-Orozco, C. (2007). Understanding and responding to the needs of newcomer immigrant youth and families. The Prevention Researcher, 14(4), 10- 13. Retrieved from:

http://educ606.wikispaces.com/file/view/Understanding+and+Respondin g.pdf

Clough, I. 2017. The informal faces of the (neo-)ghetto: State confinement, formalization and multidimensional informalities in Italy's Roma camps. International Sociology, Vol. 32(4), pp. 545-562.

Craig, S. E. (2008). Reaching and teaching children who hurt: Strategies for your classroom. Baltimore, MD:Paul H. Brookes Pub.

Crandall, J. A., Bernache, C., \& Prager, S. (1998). New frontiers in educational policy and program development: The challenge of the underschooled secondary school immigrant student. Educational Policy, 12 (6), pp.719-734. doi:10.1177/0895904898012006007

Crandall, J., \& Greenblatt, L. (1998). Teaching beyond the middle: meeting the needs of underschooled and high-achieving immigrant students. En Basterra, Excellence and Equality for Language Minirity Students: 
Critical Issues and Promising Practices. Chavy Chase: The Mid-Atlantic Equity Center.

DeCapua, A., \& Marshall, H.W. (2011). Reaching ELLs at risk: Instruction for students with limited or interrupted formal education. Preventing School Failure, 55 (1), pp. 35-41. doi: 10.1080/10459880903291680

DeCapua, A., \& Marshall, H. W. (2010). Serving ELLs with limited or interrupted education: Intervention that works. TESOL Journal, 1(1), pp. 49-70. doi:10.5054/tj.2010.214878

DeCapua, A., Smathers, W., \& Tang, L. (2007). Schooling, interrupted. Educational Leadership, 64(6), pp. 40-46. Retrived from: https://www.academia.edu/5551267/Schooling-Interrupted

DiCerbo, P., \& Loop, C. (2003) Interrupted formal schooling. National clearinghouse for English language acquisition Toolkit, 2003.Retrieved from http://www.colorincolorado.org/article/how-support-ell-studentsinterrupted-formal-education-sifes

Flecha, R., Soler-Gallart, M., \& Sordé, T. (2015). Social impact: Europe must fund social sciences. Nature, 528(7581), p. 193. doi:101038/528193d

Fleishman, H.L., \& Hospstock, P.J. (1993). Descriptive study of services to limited English proficient students. Arlington: Development Associates. Freeman, Y. S., Freeman, D. E., \& Mercuri, S. (2002). Keys to success for bilingual students with limited formal schooling. Bilingual Research Journal, 25(1), pp. 203-213. doi:10.1080/15235882.2001.10162790

Fry, R. (2003). Hispanic youth dropping out of U.S. schools: Measuring the challenge. Washington, DC: Pew Hispanic Center.

García, O. (1999). Educating Latino high school students with little formal schooling. In So Much to Say. Adolescents, bilingualism and ESL in the Secondary School, ed by Christian Faltis and Paula Wolfe. New York: Teachers College Press, pp. 61-82.

García, O. (2009). Emergent bilinguals and TESOL. What's in a name. TESOL Quaterly , 43 (2), pp. 322-326. doi:10.1002/j.15457249.2009.tb00172.x/full

Girbés-Peco, S., Macías, F., \& Álvarez, P. (2015). De la escuela gueto a una comunidad de aprendizaje: Un estudio de caso sobre la superación de la pobreza a través de una educación de éxito, International and 
336 Valero \& Plaja - Educación Inclusiva del Alumnado Inmigrante

Multidisciplinary Journal of Social Sciences 4(1), pp. 88-116, doi:10.17583/rimcis.2015.1470

Hickey, P. J. (2015). Behind the acronym: Multilingual learners with interrupted formal education. English Journal, 104(6), pp. 81-83.

Retrieved from

http://search.proquest.com/docview/1693822377? accountid=14795

Indiana Department of Education-Office of English Language Learning \&

Migrant Education (2009). Effective Programs for English Language

Learners (ELL) with Interrupted Formal Education. Retrieved from:

http://www.brycs.org/documents/upload/SIFE-Article-final-OT.pdf

Johansson, L., Angst, K., Beer, B., Martin, S., Rebeck, W., \& Sibilleau, N. (2001). Canadian language benchmarks 2000: ESL for literacy learners. Ottawa, ON. Centre for Canadian Language Benchmarks.

Kanu, Y. (2008). Educational needs and barriers for African refugee students in Manitoba. Canadian Journal of Education 31(4), 915-940. Retrived from: http://files.eric.ed.gov/fulltext/EJ830509.pdf

Klein, E., \& Martohardjono, G. (2006). Understanding the student with interrupted formal education (SIFE): A study of SIFE skills, needs and achievement. New York: New York City Department of Education.

Klein, E., \& Martohardjono, G. (2008). Investigating second language readers with low native language literacy. In American Association for Applied Linguistics $(A A A L)$ - Congreso llevado a cabo en Washington, DC.

Lee, S. J. (2012). New talk about ELL students. Phi Delta Kappan, 93(8), pp. 66-69. doi:/10.1177/003172171209300816

Lightbown, P. M., \& Spada, N. (2011). How languages are learned. Oxford: Oxford University

Mace-Matluck, B. J., Alexander-Kasparik, R., \& Queen, R. M. (1998).

Through the golden door: Educational approaches for immigrant adolescents with limited schooling. Topics in Immigrant Education 3. Language in Education: Theory and Practice 91(1). Center for Applied Linguistics, NW, Washington, DC 20037-1214. Retrived from: http://files.eric.ed.gov/fulltext/ED421019.pdf 
Matthews, J. (2008). Schooling and settlement: Refugee education in Australia. International Studies in Sociology of Education 18(1), pp. 3145. doi: 10.1080/09620210802195947

Medina Audelo, R. (2017). La construcción sociodiscursiva de la identidad del inmigrante latinoamericano en Barcelona. Social and Education History 6(2), pp. 116-141. doi:10.17583/hse.2017.2399

Menken, K. (2013). Emergent bilingual students in secondary school: Along the academic language and literacy continuum. Language Teaching, 46 (4), pp. 438-476. doi: 10.1017/S0261444813000281

New York State Department of Education. (2011). Guidelines for educating limited English proficient students with interrupted formal education. Retrieved from http://www.nysed.gov/program-offices/office-bilingualeducation-and-world-languages-obewl/docs/NYSEDSIFEGuidelines.pdf

OECD (2015). Education at a Glance 2015; OECD Indicators: Indicator D1. OECD Publishing.

Olsen, L., \& Jaramillo, A. (1999). Turning the tides of exclusion: A guide for educators and advocates for immigrant students. Oakland: California Tomorrow

Olsen, L., Jaramillo, A., Mc-Call-Perez, Z., \& White, J. (1999). Igniting change for immigrants students: Portraits of three high schools. Oakland , California: California Tomorrow.

Robertson, K., \& Lafond, S. (2008). How to support ELL students with interrupted formal education. Retrieved from

http://www.colorincolorado.org/article/how-support-ell-studentsinterrupted-formal-education-sifes

Ruíz de Velasco, J., Fix, M., \& Chu Clewell, B. (2000). Overlooked \& underserved: Immigrant students in US secondary schools. Washington D.C.: The Urban Institute.

Short, D. (2002). Newcomer programs: An educational alternative for secondary immigrant students. Education and Urban Society, 34(1), pp.173-198. doi:10.1177/0013124502034002004

Tellado, I. (2017). Bridges between individuals and communities: dialogic participation fueling meaningful social engagement. Research on Ageing and Social Policy, 5(1), pp.8-31. doi:10.17583/rasp.2017.2389 
Thomas, A. (2004). Meeting the needs of secondary ESL learners with disrupted schooling - Planning bridging programs. Victoria Department of Education and Training. Retrieved from

http://www.education.vic.gov.au/school/teachers/support/Pages/default.as px

Toohey, K., \& Derwing, T. M. (2008). Hidden losses: How demographics can encourage incorrect assumptions about ESL high school students' success. Alberta Journal of Educational Research,54(2), pp.178-193. Retrieved from

http://ajer.journalhosting.ucalgary.ca/index.php/ajer/article/view/628/611

UNESCO, UIS. Global survey on instructional time 2012. UNESCO International Bureau of Education (UNESCO-IBE). Retrieved from http://www.documan.net/d/UIS-Survey-on-Statistics-of-Informationand.pdf

Woods, A. (2009). Learning to be literate: Issues of pedagogy for recently arrived refugee youth in Australia. Critical Inquiry in Language Studies, 6(1-2) pp.82-101. doi:10.1080/15427580802679468

Wright, E.W. (2010). Foundations for teaching English language learners: Research, theory, policy and practice. Philadelphia, PA: Caslon Publishing.

Diana Valero es investigadora post-doctoral en la Universidad de Zaragoza

Teresa Plaja Viñas es investigadora predoctoral en la Universidad de Barcelona

Dirección de Contacto: Calle Albareda, 7, 2 - 2ª , 50004 Zaragoza E-mail: dvalero@unizar.es 